\title{
A practical guide to unravel time-like transition form factors
}

\author{
Simone Pacetti \\ Enrico Fermi Center, Rome, Italy \\ INFN, Laboratori Nazionali di Frascati, Frascati, Italy \\ E-mail: simone.pacetti@lnf.infn.it
}

\begin{abstract}
A method to determine masses, widths and coupling constants of vector mesons, like $\phi(1020), \omega(782)$ and $\rho^{0}(770)$ recurrences is defined. Starting from data on decay rates and cross sections for the processes: $\phi \rightarrow M_{I} \gamma, \phi \rightarrow M_{I} e^{+} e^{-}$and $e^{+} e^{-} \rightarrow M_{I} \phi$, where $M_{I}$ is a pseudoscalar or scalar meson with isospin $I=0,1$, the time-like transition form factors, which describe the vertex $\phi \gamma M_{I}$, are parametrized using a vector mesonpropagators description in the low energy region $(<3-4 \mathrm{GeV})$, the quark-counting rule prescription for the high energy behavior, and the analyticity imposed by means of the dispersion relations.
\end{abstract}




\section{Contents}

1. Introduction

1.1 "Static" form factors

1.2 "Dynamic" time-like form factors

2. Strategy 6

2.1 Extracting the transition form factor from data

2.2 Parameterization in the resonance region 9

2.3 Selection of the resonant contributions 10

2.3.1 Isoscalar $M_{I=0}$

2.3.2 Isovector $M_{I=1}$

2.4 The asymptotic behavior 13

2.5 Analytic continuation in the $q^{2}$-complex plane 15

2.6 The overall parameterization 15

3. $\chi^{2}$ definition 16

3.1 Free parameters and fixed values $\quad 16$

3.2 Constraints 17

$3.3 \chi^{2}$ expression 17

4. Possible aims 18

4.1 Looking for masses, widths and coupling constants 18

4.2 Investigating quark structures 18

5. Conclusion 18

\section{Introduction}

Recently the interest in the electromagnetic hadron structures has increased considerably due to the large amount of data coming from both flavor factories [1], working with initial state radiation (ISR), and fixed-target machines operating with polarized beams [2].

The study of phenomenological properties of hadrons: the magnetic moments, structure functions, number, species and mixing of quarks and gluons in their wave functions, etc. plays a fundamental role in our understanding of the QCD dynamics. The analysis of electromagnetic decays and hadron-photon interactions provides unique information for this purpose. Indeed, since the photon-hadron coupling occurs through the direct interaction of the photon with the electric charges of single quarks, such processes represent probes by the intrinsic structure of hadrons. 


\section{1 "Static" form factors [3]}

The first attempt to unravel the electromagnetic structure of a hadron by studying a form factor (ff) was done by Rutherford in his famous experiment. In that case he studied the scattering of a charged probe, the ${ }^{4} \mathrm{He}$ nucleus, in the electromagnetic field of atoms of gold. The intensity of the scattered ${ }^{4} \mathrm{He}$ beam as a function of the scattering angle was just a measure of the electromagnetic $\mathrm{ff}$ as a function of the transferred momentum.
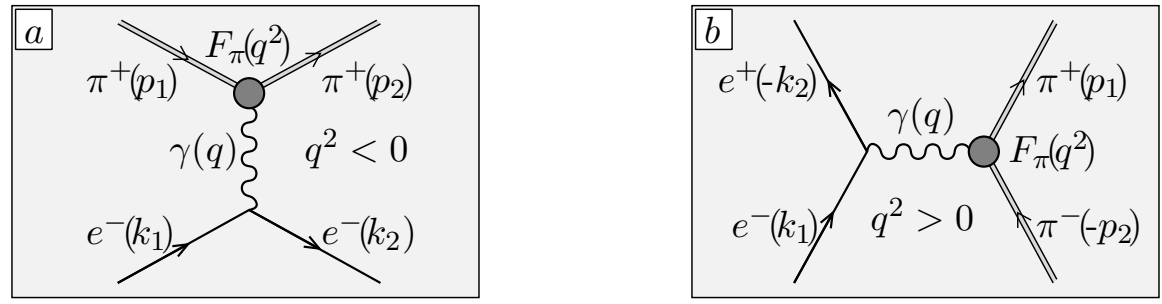

Figure 1: One-photon exchange diagrams: for the scattering $e^{-} \pi^{+} \rightarrow e^{-} \pi^{+}(a)$ and for the annihilation $e^{+} e^{-} \rightarrow \pi^{+} \pi^{-}(b)$.

We consider in more details the photon-pion interaction, i.e. the vertex $\pi \gamma \pi$ shown in fig. 1 , in case of scattering $e^{-} \pi^{+} \rightarrow e^{-} \pi^{+}$(fig. [ $1 a$ ) and annihilation $e^{+} e^{-} \rightarrow \pi^{+} \pi^{-}$(fig. 1 $1 b$ ).

For pointlike particles, as the leptons, the electromagnetic current which describes the vertex eqe (fig. 1 $1 a, b$ ) has the form $J_{e}^{\mu}=-i e \bar{u}\left(k_{2}\right) \gamma^{\mu} u\left(k_{1}\right)$ (with 4-momenta as labeled in fig. 1). The electromagnetic current, in the case of the hadronic vertex $\pi \gamma \pi$, is

$$
J_{\pi}^{\mu}=\underbrace{i e\left(p_{1}+p_{2}\right)^{\mu}}_{\text {pointlike }} F_{\pi}\left(q^{2}\right)
$$

where $p_{1}$ and $p_{2}$ are the 4 -momenta (see fig. 目), and $F_{\pi}\left(q^{2}\right)$, the pion ff, is a scalar function of the transferred momentum squared. The tensor part of this current, called "pointlike" in expression (1.1), is achieved by requiring that the most general tensorial form, which derives from the spin properties of the particles under consideration, fulfills Lorentz and gauge invariance ${ }^{1}$. The ff gives an exhaustive characterization of the spatial distribution of charge of the pion, i.e. it accounts for the extended hadronic structure of the particle. In the nonrelativistic limit, the ff tends to the Fourier transformation of the charge density distribution.

For high-energy photon-hadron interactions the relation with the charge density becomes more complex than the simple Fourier transformation. In spite of that, the ff describes unambiguously the electromagnetic structure of the particle and it represents the only quantity, intrinsically related to this structure, which is experimentally accessible.

Using the previous expressions for the leptonic and hadronic currents, the differential cross section for the scattering $e^{-} \pi^{+} \rightarrow e^{-} \pi^{+}$, in Born approximation (see fig. 1), can be written as:

$$
\frac{d \sigma\left(e^{-} \pi^{+} \rightarrow e^{-} \pi^{+}\right)}{d q^{2}}=\left[\frac{d \sigma\left(e^{-} \pi^{+} \rightarrow e^{-} \pi^{+}\right)}{d q^{2}}\right]_{\text {pointlike }}\left[F_{\pi}\left(q^{2}\right)\right]^{2}
$$

${ }^{1}$ Charge conjugation invariance requires a vanishing neutral pion $\mathrm{ff}$. 
The values of the ff may be extracted by comparing cross section data and the differential cross section computed in the case of pointlike charged hadrons, $\left[d \sigma / d q^{2}\right]_{\text {pointlike }}$. By using the scattering cross section data, only space-like values can be investigated. In this case $q^{2}=\left(p_{1}-p_{2}\right)^{2} \leq 0$. In the space-like region the $\mathrm{ff}$ is a real function, hence the data on the differential cross section $\frac{d \sigma\left(e^{-} \pi^{+} \rightarrow e^{-} \pi^{+}\right)}{d q^{2}}$ represent a direct measurement of the ff itself. The annihilation $e^{+} e^{-} \rightarrow \pi^{+} \pi^{-}$, shown in fig. $1 b$ in case of one-photon exchange, is the scattering crossed process. It contains the same vertices and the cross section, computed by contracting the same currents with opposite signs for antiparticle 4-momenta, reads:

$$
\sigma\left(e^{+} e^{-} \rightarrow \pi^{+} \pi^{-}\right)=\left[\sigma\left(e^{+} e^{-} \rightarrow \pi^{+} \pi^{-}\right)\right]_{\text {pointlike }}\left|F_{\pi}\left(q^{2}\right)\right|^{2} .
$$

In this case $q^{2}=\left(p_{1}-p_{2}\right)^{2} \geq 4 M_{\pi}^{2}$, so by measuring the annihilation cross section we are probing the $\mathrm{ff}$ in the portion of the time-like region above the physical threshold $4 M_{\pi}^{2}$. Here the $\mathrm{ff}$ is a complex function and only its modulus is experimentally accessible [see expression (1.3)]. No information about the complex structure of this function, i.e. real and imaginary parts, may be obtained by direct experimental observation.

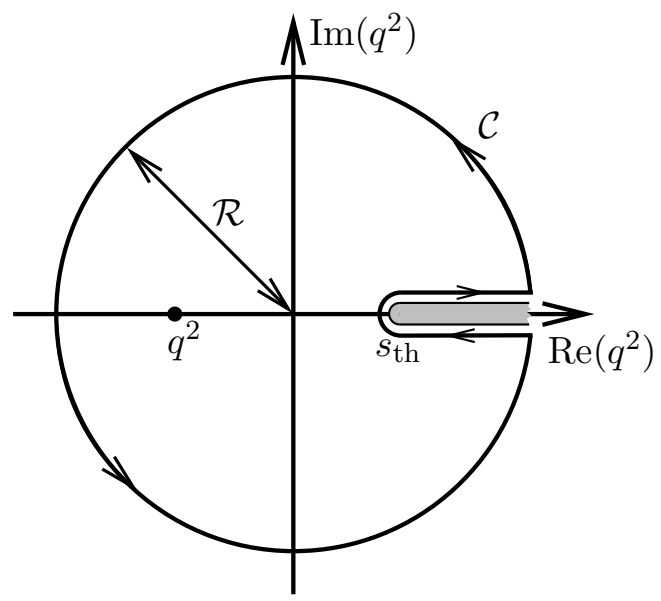

Figure 2: Analyticity domain for the pion ff. The shadowed band indicates the cut over the real axis. The space-like $q^{2}$, on the left, is a generic point where the real values of the ff may be extracted from the scattering cross section.

Nevertheless, by invoking unitarity and analyticity for the ff's, integral relations between the modulus and the phase above threshold may be established. By means of these relations, the phase is computed as a function of the modulus and therefore we gain a complete knowledge of the complex structure of the ff's.

In general (see fig. 2), the ff's are analytic functions in the $q^{2}$-complex plane with a cut, on the real axis, starting from the theoretical threshold $s_{\text {th }}=4 M_{\pi}^{2}$, which, in this case, corresponds to the physical one, up to infinity. We introduce here the concepts of theoretical and physical threshold for a ff. Both of them lie in the time-like region and refer to the annihilation process.

- The theoretical threshold, called $s_{\mathrm{th}}$, is the energy of the first hadronic channel that carries the same quantum numbers of the final state under consideration, e.g. in this 
case $\pi^{+} \pi^{-}$, produced through the $e^{+} e^{-}$annihilation.

- The physical threshold corresponds to the production energy of the hadronic final state, it is greater or, only in few cases, equal to the physical one.

The pion $\mathrm{ff}$ is just one of these special cases where the thresholds correspond to the same energy. The discontinuity over the real axis is the superposition of infinite cuts, each of which corresponds to the opening of a channel for every allowed final state. In principle these functions are well defined for all complex $q^{2}$ outside the cut, but only real values of $q^{2}$ are experimentally accessible.

The case of the pion $\mathrm{ff}$ is particularly simple, mainly due to the fact that it is a boson with spin zero. The study of nucleon ff's requires a more complicated treatment. These particles are fermions and their spin structures are described by two, instead of only one, ff's. Nevertheless, the scheme and the method of the analysis remain the same.
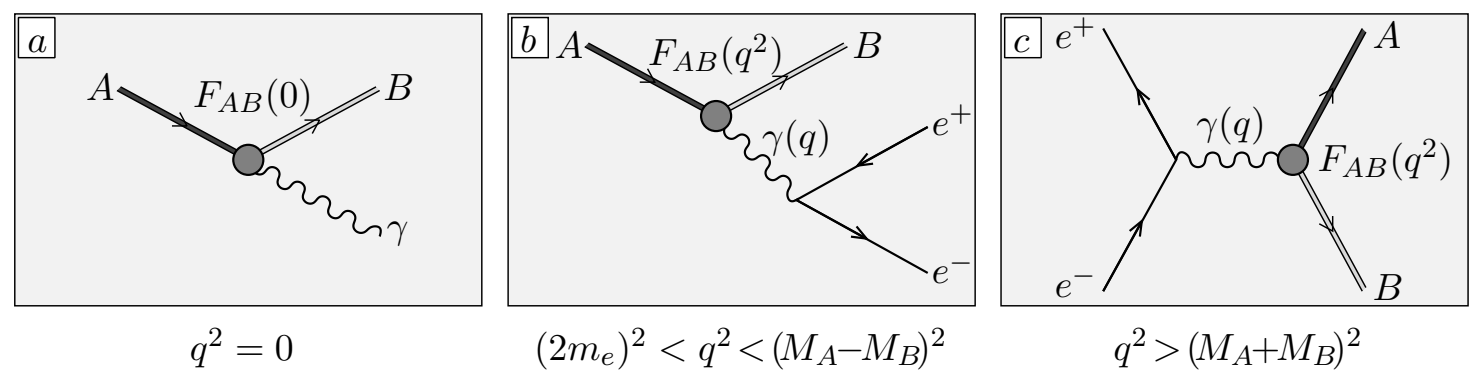

Figure 3: Diagrams for the radiative decay $A \rightarrow B \gamma(a)$, the conversion decay $A \rightarrow B e^{+} e^{-}(b)$ and annihilation $e^{+} e^{-} \rightarrow A B(c)$. In all these cases the coupling $A \gamma B$ is described by the same tff $F_{A B}\left(q^{2}\right)$, but evaluated in the different regions of $q^{2}$.

\section{2 "Dynamic" time-like form factors [3]}

As we have already stated the ff's provide a crucial mean to investigate the internal hadron structure. They describe the photon-hadron interaction in terms of coupling between photons and charges of quark constituents, and hence they give unique information about the QCD dynamics inside a hadron. However, up to now we have dealt with only "static" structure functions, i.e. ff's that, due to the charge conjugation invariance ${ }^{2}$, are defined only for charged mesons. To study the internal structure of neutral hadrons, we may consider vertices like $A \gamma B$, where a neutral particle $A$ converts into a different neutral particle $B$ with the emission of a photon while conserving all the quantum numbers. The physical process would be (see fig. 嗮):

$$
A \rightarrow B+\gamma, \quad \text { charge conjugation conservation } \Longrightarrow C_{A}=C_{B} \cdot C_{\gamma},
$$

where, to conserve charge conjugation, being $C_{\gamma}=-1$, we have: $C_{A}=-C_{B}$. For every pair of neutral mesons for which the radiative decay (1.4) is allowed, these other one-photon exchange processes also are allowed:

\footnotetext{
${ }^{2}$ One-photon exchange processes (see fig. 1) for neutral mesons have vanishing amplitude, and then vanishing ff for all values of $q^{2}$, since the charge-conjugation parity is conserved in electromagnetic interactions.
} 
- conversion decay (see fig. 3b): $A \rightarrow B \gamma^{*} \rightarrow B e^{+} e^{-}$with $\left(2 m_{e}\right)^{2}<q^{2}<\left(M_{A}-M_{B}\right)^{2}$ ( $m_{e}$ is the electron mass);

- annihilation (see fig. 3c): $e^{+} e^{-} \rightarrow \gamma^{*} \rightarrow A B$ with $q^{2} \geq\left(M_{A}+M_{B}\right)^{2}$.

The probability for the meson $A$ to convert into a meson $B$ by emitting a photon of 4 momentum $q$ is described by a specific ff called the "dynamic" or transition form factor (tff) $F_{A B}\left(q^{2}\right)$. Contrary to the ordinary "static" ff's which, being related to the structure of a single particle, vanish for neutral mesons, the tff's, that describe the electromagnetic structure of transition vertex, i.e. the dynamics of the conversion of a meson into another, are, in principle, different from zero. To extract values of a tff from experimental data on decay rates and annihilation cross section we define the dynamic conversion current:

$$
J_{A B}^{\mu}=\mathcal{J}_{\text {pointlike }}^{\mu}\left(q^{2}\right) \cdot F_{A B}\left(q^{2}\right),
$$

where the tensor $\mathcal{J}_{\text {pointlike }}^{\mu}\left(q^{2}\right)$, obtained by requiring Lorentz and gauge invariance, describes the vertex $A \gamma B$ in case of structureless particles and the tff $F_{A B}\left(q^{2}\right)$ accounts for the modifications of this vertex due to the extended electromagnetic internal structures of the particles $A$ and $B$.

In more detail, to investigate the tff $F_{A B}\left(q^{2}\right)$, three experimental observables may be considered:

- the radiative decay rate that, by using the current (1.5), has the form:

$$
\Gamma(A \rightarrow B \gamma)=\mathcal{G}_{\text {pointlike }} \cdot\left[F_{A B}(0)\right]^{2},
$$

this is a constant quantity which depends on the real value of the tff at $q^{2}=0$ (real photon).

- The differential decay rate:

$$
\frac{d \Gamma\left(A \rightarrow B e^{+} e^{-}\right)}{d q^{2}}=\left[\frac{d \mathcal{G}}{d q^{2}}\right]_{\text {pointlike }} \cdot\left|F_{A B}\left(q^{2}\right)\right|^{2},
$$

with: $\left(2 m_{e}\right)^{2}<q^{2}<\left(M_{A}-M_{B}\right)^{2}$.

- The annihilation cross section:

$$
\sigma\left(e^{+} e^{-} \rightarrow A B\right)=\mathcal{S}_{\text {pointlike }} \cdot\left|F_{A B}\left(q^{2}\right)\right|^{2},
$$

with: $q^{2}>\left(M_{A}+M_{B}\right)^{2}$.

The quantities $\mathcal{G}_{\text {pointlike }},\left[d \mathcal{G} / d q^{2}\right]_{\text {pointlike }}$, and $\mathcal{S}_{\text {pointlike }}$ represent the kinematic factors obtained using the current (1.5) with $F_{A B}\left(q^{2}\right)=1$. As we will see in the following [s2.1, eq. (2.8)], an interpretation in terms of pointlike decay rates and annihilation cross section of these quantities, obtained by using only the pointlike part of the current (1.5), appears awkward because of the wrong dimensions.

Finally we note that, although the tff's, exactly as the ff's, are analytic functions defined 
in the whole $q^{2}$-complex plane with the cut $\left(s_{\mathrm{th}}, \infty\right)$, the listed processes cover only a portion of the time-like region. In principle the space-like region could be investigated by measuring the "conversion scattering" $A e^{-} \rightarrow B e^{-}$, however the enormous difficulty in realizing a stable massive meson beam, makes this possibility a very hard task. In the time-like region there is an interval which is theoretically not accessible by the experiments, it is the so-called "unphysical" region: $\left[\left(M_{A}-M_{B}\right),\left(M_{A}+M_{B}\right)\right]$.

In the rest of this work we will focus on a particular group of tff's, i.e. those describing the $\phi(1020) \gamma M$ conversion, where $\phi(1020)$ is $s \bar{s}$ vector meson and $M$ is a generic light (made of $u, d$, and $s$ quarks) pseudoscalar or scalar meson.

\section{Strategy}

We define a general procedure to study the tff of a generic conversion $\phi \gamma M$, where, from now on, $\phi$ stands for the vector meson $\phi(1020)$ and $M$ for a light pseudoscalar or scalar meson. By assuming that in a certain $q^{2}$ interval, called the resonance region, the photon couples with the mesons $\phi$ and $M$ through a series of intermediate vector mesons, we parametrize the tff in this region as a sum of propagators weighted by the corresponding coupling constants. This parameterization is then extended, to higher values of $q^{2}$ using the power law asymptotic behavior provided by the quark-counting rule (QcR) [4], and to all the other values of $q^{2}$ by means of a rigorous analytic continuation technique, based on dispersion relations. The free parameters of such a description, which cover in principle the whole $q^{2}$-complex plane, are determined by imposing theoretical and experimental constraints.

\subsection{Extracting the transition form factor from data}

To extract experimental values of the tff's, data on decay rates and cross section are compared with the kinematic factors computed in the case of constant couplings, i.e. pointlike mesons [eqs. (1.6 1.8)]. The explicit forms for the conversion currents needed to compute the kinematic factors in the cases: $M=\operatorname{pseudoscalar}(P)$ and $M=\operatorname{scalar}(S)$, are [eq. (1.5)]:

$$
\begin{aligned}
\mathcal{J}_{\phi P}^{\mu} & =e \epsilon^{\mu \nu \rho \sigma} \varepsilon_{\nu} p_{\rho} q_{\sigma} \\
J_{\phi M}^{\mu}=\mathcal{J}_{\phi M}^{\mu} \cdot F_{\phi M}(s) & \mathcal{J}_{\phi S}^{\mu}=e\left[p^{\mu} q^{\nu}-g^{\mu \nu}(p q)\right] \varepsilon_{\nu}
\end{aligned},
$$

where $\epsilon^{\mu \nu \rho \sigma}$ is the fully antisymmetric Levi-Civita tensor and, following the labeling of fig. औ, $q$ is the 4-momentum of the photon and, $p$ and $\varepsilon$ are the 4-momentum and the polarization vector of the $\phi$.

The structure of the tensors which describe the dynamics of the pseudoscalar (scalar) meson in the vertex $\phi \gamma P(S)$, reported in eq. (2.1), follows from general arguments, i.e. spin properties, Lorentz and gauge invariance. In particular, we note that in both cases, only one tff is needed to describe the conversion.

Once the conversion currents have been defined, rates and cross section may be computed. 


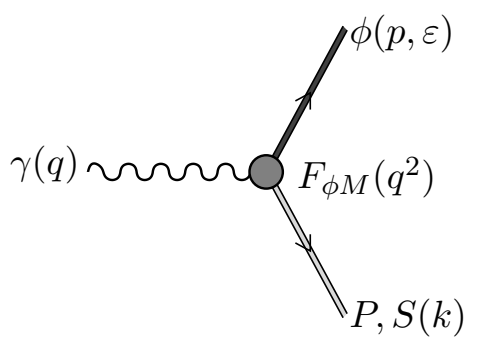

Figure 4: Diagram for the conversion $\phi \gamma P, S$.

First, we consider the radiative decay rates: $\phi(p) \rightarrow M(k) \gamma(q)$ shown in fig. 3a with $A \equiv \phi$ and $B \equiv M=P, S$, the Feynman amplitudes read:

$$
\begin{aligned}
& \mathcal{M}(\phi \rightarrow P \gamma)=e F_{\phi P}(0) \epsilon^{\mu \nu \rho \sigma} \varepsilon_{\nu} p_{\rho} q_{\sigma}{\varepsilon^{\prime}}_{\mu}^{*} \\
& \mathcal{M}(\phi \rightarrow S \gamma)=e F_{\phi S}(0)\left[p^{\mu} q^{\nu}-g^{\mu \nu}(p q)\right] \varepsilon_{\nu} \varepsilon_{\mu}^{\prime *}
\end{aligned}
$$

where $\varepsilon^{\prime}$ is the photon polarization vector. Both these amplitudes depend on the value of the tff at $q^{2}=0$ because the photon is real. From these, we calculate the radiative decay rates:

$$
\begin{gathered}
\Gamma(\phi \rightarrow P \gamma)=\frac{\alpha}{3}\left(\frac{M_{\phi}^{2}-M_{P}^{2}}{2 M_{\phi}}\right)^{3}\left[F_{\phi P}(0)\right]^{2} \\
\Gamma(\phi \rightarrow S \gamma)=\frac{\alpha}{3}\left(\frac{M_{\phi}^{2}-M_{S}^{2}}{2 M_{\phi}}\right)^{3}\left[F_{\phi S}(0)\right]^{2} .
\end{gathered}
$$

The kinematic factors are exactly the same, hence, at $q^{2}=0$, the different natures of the mesons are not kinematically distinguishable.

The amplitudes for the conversion decays $\phi(p) \rightarrow P, S(k) e^{+}\left(p_{+}\right) e^{-}\left(p_{-}\right)$in the Born approximation, shown in fig. $3 b(A \equiv \phi$ and $B \equiv M=P, S)$, are:

$$
\begin{aligned}
& \mathcal{M}\left(\phi \rightarrow P e^{+} e^{-}\right)=-i e^{2}\left[F_{\phi P}\left(q^{2}\right) \epsilon^{\mu \nu \rho \sigma} \varepsilon_{\nu} p_{\rho} q_{\sigma}\right] \frac{1}{q^{2}}\left[\bar{u}\left(p_{-}\right) \gamma_{\mu} v\left(p_{+}\right)\right] \\
& \mathcal{M}\left(\phi \rightarrow S e^{+} e^{-}\right)=-i e^{2}\left\{F_{\phi S}\left(q^{2}\right)\left[p^{\mu} q^{\nu}-g^{\mu \nu}(p q)\right] \varepsilon_{\nu}\right\} \frac{1}{q^{2}}\left[\bar{u}\left(p_{-}\right) \gamma_{\mu} v\left(p_{+}\right)\right],
\end{aligned}
$$


with: $\left(2 m_{e}\right)^{2}<q^{2}<\left(M_{\phi}-M_{M}\right)^{2}$, and the corresponding differential decay rates:

$$
\begin{aligned}
\frac{d \Gamma\left(\phi \rightarrow P e^{+} e^{-}\right)}{d q^{2}}= & \frac{\alpha^{2}}{9 \pi} \frac{1}{\left(2 M_{\phi}\right)^{3}} \sqrt{1-\frac{4 m_{e}^{2}}{q^{2}}}\left(1+\frac{2 m_{e}^{2}}{q^{2}}\right) \frac{1}{q^{2}} \times \\
& \times\left[\left(q^{2}+M_{\phi}^{2}-M_{P}^{2}\right)^{2}-4 M_{\phi}^{2} q^{2}\right]^{\frac{3}{2}}\left|F_{\phi P}\left(q^{2}\right)\right|^{2} \\
\frac{d \Gamma\left(\phi \rightarrow S e^{+} e^{-}\right)}{d q^{2}}= & \frac{\alpha^{2}}{9 \pi} \frac{1}{\left(2 M_{\phi}\right)^{3}} \sqrt{1-\frac{4 m_{e}^{2}}{q^{2}}}\left(1+\frac{2 m_{e}^{2}}{q^{2}}\right) \frac{1}{q^{2}} \times \\
& \times\left[\left(q^{2}+M_{\phi}^{2}-M_{S}^{2}\right)^{2}+2 M_{\phi}^{2} q^{2}\right]\left[\left(q^{2}+M_{\phi}^{2}-M_{S}^{2}\right)^{2}-4 M_{\phi}^{2} q^{2}\right]^{\frac{1}{2}}\left|F_{\phi S}\left(q^{2}\right)\right|^{2} .
\end{aligned}
$$

In this $q^{2}$ region the different natures of the meson $M$ provide different kinematic structures. Finally we consider the annihilation process $e^{+}\left(p_{+}\right) e^{-}\left(p_{-}\right) \rightarrow \phi(p) P, S(k)$, shown in fig. 3$] c$ $(A \equiv \phi$ and $B \equiv M=P, S)$ in the Born approximation. The amplitudes are:

$$
\begin{aligned}
& \mathcal{M}\left(e^{+} e^{-} \rightarrow \phi P\right)=-i e^{2}\left[F_{\phi P}\left(q^{2}\right) \epsilon^{\mu \nu \rho \sigma} \varepsilon_{\nu} p_{\rho} q_{\sigma}\right] \frac{1}{q^{2}}\left[\bar{v}\left(p_{+}\right) \gamma_{\mu} u\left(p_{-}\right)\right] \\
& \mathcal{M}\left(e^{+} e^{-} \rightarrow \phi S\right)=-i e^{2}\left\{F_{\phi S}\left(q^{2}\right)\left[p^{\mu} q^{\nu}-g^{\mu \nu}(p q)\right] \varepsilon_{\nu}\right\} \frac{1}{q^{2}}\left[\bar{v}\left(p_{+}\right) \gamma_{\mu} u\left(p_{-}\right)\right],
\end{aligned}
$$

for $q^{2}>\left(M_{\phi}+M_{M}\right)^{2}$ and the cross sections:

$$
\begin{aligned}
\sigma\left(e^{+} e^{-} \rightarrow \phi P\right)= & \frac{\pi}{6} \frac{\alpha^{2}}{\left(q^{2}\right)^{3}} \frac{q^{2}+2 m_{e}^{2}}{\sqrt{q^{2}\left(q^{2}-4 m_{e}^{2}\right)}} \times \\
& \times\left[\left(q^{2}+M_{\phi}^{2}-M_{P}^{2}\right)^{2}-4 M_{\phi}^{2} q^{2}\right]^{\frac{3}{2}}\left|F_{\phi P}\left(q^{2}\right)\right|^{2} \\
\sigma\left(e^{+} e^{-} \rightarrow \phi S\right)= & \frac{\pi}{6} \frac{\alpha^{2}}{\left(q^{2}\right)^{3}} \frac{q^{2}+2 m_{e}^{2}}{\sqrt{q^{2}\left(q^{2}-4 m_{e}^{2}\right)}} \times \\
& \times\left[\left(q^{2}+M_{\phi}^{2}-M_{S}^{2}\right)^{2}+2 M_{\phi}^{2} q^{2}\right]\left[\left(q^{2}+M_{\phi}^{2}-M_{S}^{2}\right)^{2}-4 M_{\phi}^{2} q^{2}\right]^{\frac{1}{2}}\left|F_{\phi S}\left(q^{2}\right)\right|^{2} .
\end{aligned}
$$

Once again the pseudoscalar and scalar natures of the meson $M$ are reflected in the different kinematic factors that have to be considered to extract tff's from data.

It is interesting to note that the dimension of the tensors (2.1) is not that of a current, i.e. energy. It follows that the tff's, unlike static ff's, are dimensional quantities. In particular, we have:

$$
\left[\mathcal{J}_{\phi M}^{\mu}\right]=E^{2} \quad \Longrightarrow \quad\left[F_{\phi M}\right]=E^{-1}
$$

This is why, when we are dealing with tff's, the kinematic factor which multiplies the tff itself to give the physical quantities: decay rates or cross sections, cannot be interpreted as the pointlike physical quantities. To get pointlike quantities we must replace the tff's with dimensional $\left(E^{-1}\right)$ coupling constants. Such a replacement should provide an unusual 
result in case of annihilation cross section. If we consider the formulae of eq. (2.7), where we put $F_{\phi M}\left(q^{2}\right) \equiv g_{\phi M}^{\gamma}\left(g_{\phi M}^{\gamma}\right.$ is a constant coupling with: $\left.\left[g_{\phi M}^{\gamma}\right]=E^{-1}\right)$, in the limit $q^{2} \rightarrow \infty$, we get:

$$
\begin{gathered}
\sigma\left(e^{+} e^{-} \rightarrow \phi P\right) \underset{q^{2} \rightarrow \infty}{\longrightarrow} \frac{\pi \alpha^{2}}{6}\left|g_{\phi P}^{\gamma}\right|^{2} \\
\sigma\left(e^{+} e^{-} \rightarrow \phi S\right) \underset{q^{2} \rightarrow \infty}{\longrightarrow} \frac{\pi \alpha^{2}}{6}\left|g_{\phi S}^{\gamma}\right|^{2} .
\end{gathered}
$$

Constant asymptotic limits, in case of pointlike mesons, represent an unexpected result. The usual "minimal" expectation is a behavior that mimics the cross section $\sigma\left(e^{+} e^{-} \rightarrow\right.$ $\left.\mu^{+} \mu^{-}\right)$which vanishes like $\left(1 / q^{2}\right)$ as $q^{2}$ diverges. However, such expected behavior is true only in case of the static ff where we have nonvanishing amplitudes only in the case of charged mesons. Since we are dealing with pairs of different neutral mesons which convert from one in to the other and since, even for "pointlike" mesons, we must consider the quark structure of these particles which allows the conversion (dynamic ff's), then additional effects providing further attenuation, must be accounted for. As we will see in the following, attenuation factors in the asymptotic regime are provided by the hadronic helicity rule [4].

\subsection{Parameterization in the resonance region}

The resonance region covers the portion of the time-like region where the tff is characterized by the vector meson resonance contributions. In general this interval goes from the theoretical threshold $s_{\text {th }}$ up to $s_{\text {asy }} \sim(3-4 \mathrm{GeV})^{2}$. Above this energy, the power law asymptotic behavior, as predicted by QcR [4], is assumed. Vector meson resonances, in the Born approximation, may be interpreted as intermediate states coupling the virtual photon and final mesons. They carry the quantum numbers of the photon, i.e. $J^{P C}=1^{--}$. In light of this, and following the definition (2.1), the conversion current $\phi \gamma M_{I}$, where $I$ is the isospin of the meson $M$, has the form:

$$
J_{\phi M_{I}}^{\mu}=\mathcal{J}_{\phi M_{I}}^{\mu} \sum_{j=1}^{N_{M_{I}}} \frac{M_{j}^{2}}{e F_{V_{j}}} \frac{g_{\phi M_{I}}^{V_{j}}}{M_{j}^{2}-q^{2}-i \Gamma_{j} M_{j}},
$$

where, as shown in fig. 5, $N_{M_{I}}$ intermediate vector mesons $V_{j}$, with mass $M_{j}$ and width $\Gamma_{j}$, are considered.

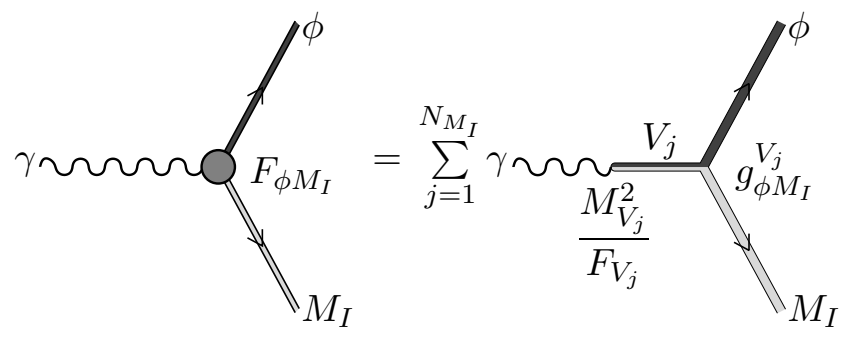

Figure 5: Schematic representation of the parameterization for tff's. 
To summarize, by assuming that the virtual photon couples with all the allowed vector mesons $V_{j}$ with a strength $\frac{M_{V_{j}}^{2}}{F_{V_{j}}}$ and then each meson $V_{j}$ goes in the final state $\phi M_{I}$ with a coupling $g_{\phi M_{I}}^{V_{j}}$, we get the tff:

$$
F_{\phi M_{I}}\left(q^{2}\right)=\sum_{j=1}^{N_{M_{I}}} \frac{M_{j}^{2}}{e F_{V_{j}}} \frac{g_{\phi M_{I}}^{V_{j}}}{M_{j}^{2}-q^{2}-i \Gamma_{j} M_{j}} \quad \quad s_{\mathrm{th}}^{I} \leq q^{2} \leq s_{\mathrm{asy}}^{M_{I}} .
$$

Note that the species which contribute to this sum and also the threshold values $s_{\text {th }}^{I}$ and $s_{\text {asy }}^{M_{I}}$ depend on the nature of the meson $M_{I}$. In particular, while $s_{\text {asy }}^{M_{I}}$ is a free parameter of the fit, $s_{\mathrm{th}}^{I}$ depends on the isospin $I$ of the meson $M_{I}$, i.e.:

$$
s_{\mathrm{th}}^{I}=\left\{\begin{array}{l}
\left(3 M_{\pi}\right)^{2} I=0 \\
\left(2 M_{\pi}\right)^{2} I=1
\end{array} .\right.
$$

This parameterization integrates all the information about the structure of the meson $M_{I}^{3}$.

\subsection{Selection of the resonant contributions}

\subsubsection{Isoscalar $M_{I=0}$}

The first criterion used to select vector meson contributions to the tff's in the resonance region, where we adopt the parameterization (2.11), is the quantum number conservation. If we consider a pseudoscalar $\left[I^{G}\left(J^{P C}\right)=0^{+}\left(1^{-+}\right)\right]$and a scalar $\left[I^{G}\left(J^{P C}\right)=0^{+}\left(0^{++}\right)\right]$ meson with isospin zero, having the $\phi$ quantum numbers: $I^{G}\left(J^{P C}\right)=0^{-}\left(1^{--}\right)$, both $\phi P_{0}$ and $\phi S_{0}$ final states (the subscript 0 indicates the isospin) will have $I^{G}\left(J^{P C}\right)=0^{-}\left(1^{--}\right)$, hence only contributions from the $\omega$ - and $\phi$-family are expected. However, by considering explicitly the structure of the mesons in terms of valence quarks, using only the light quarks $q=u, d, s$ in a $q \bar{q}$ bound state, we have:

$$
\begin{aligned}
\left|P_{0}\right\rangle & =X_{P_{0}}|u, d ;-\rangle+Y_{P_{0}}|s, s ;-\rangle \\
\left|S_{0}\right\rangle & =X_{S_{0}}|u, d ;+\rangle+Y_{S_{0}}|s, s ;+\rangle
\end{aligned}
$$

with the normalization: $X_{P_{0}, S_{0}}^{2}+Y_{P_{0}, S_{0}}^{2}=1$ and where the state $\left|q_{1}, q_{2} ; P\right\rangle$ is a normalized combination of $q_{1} \bar{q}_{1}$ and $q_{2} \bar{q}_{2}$, with parity $P$ and $J^{C}=0^{+}$. While the $\phi$-family contributions are allowed (fig. 6), the $\omega$-family contributions are instead OZI-forbidden [5]. In fact, as it is shown in fig. 7, the corresponding Feynman diagrams have disconnected flavor lines in the vector sector.

It follows that, in light of the quantum number conservation and the OZI rule, only $\phi$ family contributions are expected for both $\phi P_{0}$ and $\phi S_{0}$ final states.

Another interesting hypothesis is that the meson $M_{0}$ is a scalar tetraquark $S_{0}^{4}$ [6]. In the most stable configuration, the four quarks should arrange in diquark-antidiquark pairs $[q q][\overline{q q}]$ (in each diquark the two quarks are bound in $S$-wave to form a color and flavor

\footnotetext{
${ }^{3}$ E.g., for $M_{0} \equiv f_{0}$, a strong affinity of the $f_{0}$ with the $K \bar{K}$ intermediate state (kaon loop) should manifest itself in an enhancement of the coupling $g_{\phi f_{0}}^{\phi}$, since in the $s$-channel the $K \bar{K}$ state is almost completely resonant in $\phi(1020)$.
} 


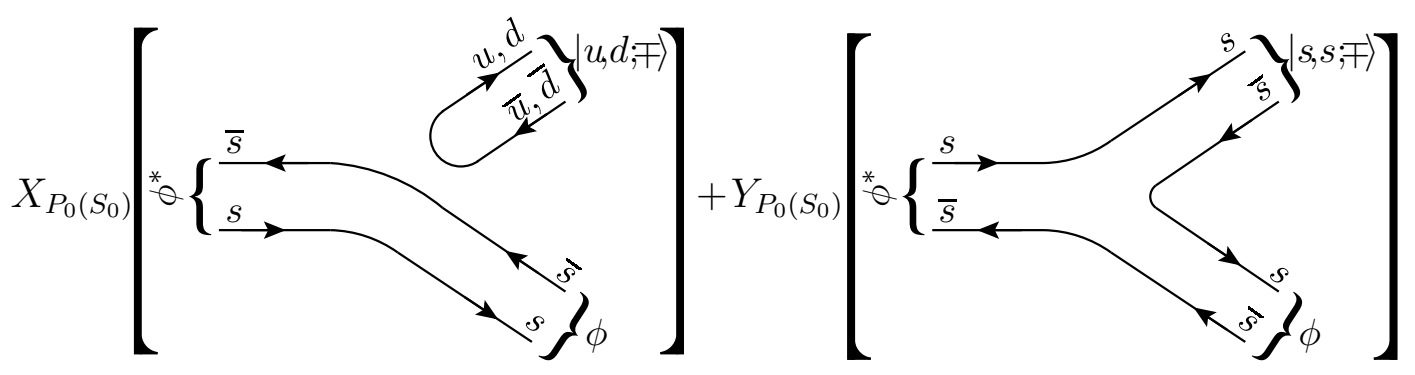

Figure 6: Feynman diagram for $\phi^{*} \rightarrow \phi P_{0}\left(S_{0}\right)$ with: $|\phi\rangle=s \bar{s}$ and $\left|P_{0}\left(S_{0}\right)\right\rangle=X_{P_{0}, S_{0}}|u, d ; \mp\rangle+$ $Y_{P_{0}, S_{0}}|s, s ; \mp\rangle$. The two components $|u, d ; \mp\rangle$ and $|s, s ; \mp\rangle$ are separately shown.

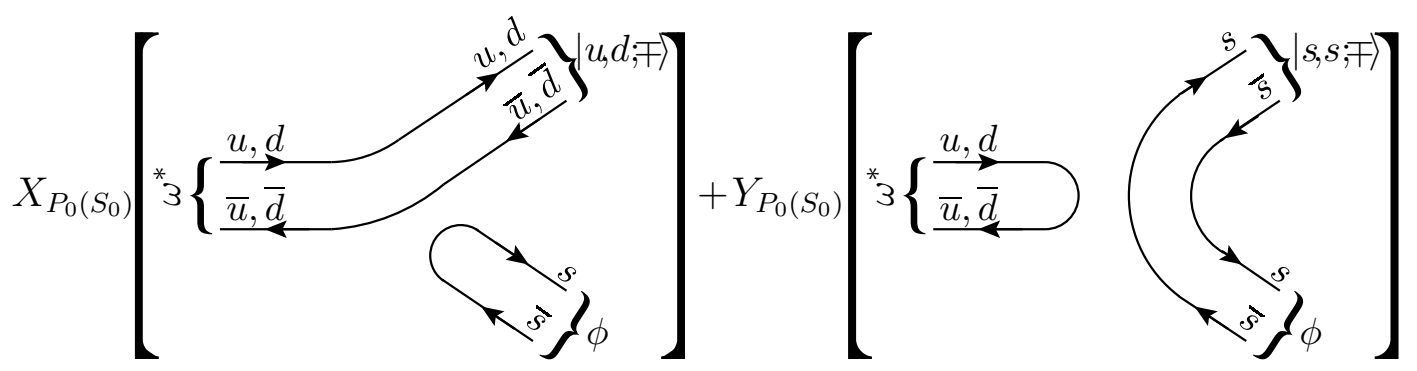

Figure 7: Feynman diagram for $\omega^{*} \rightarrow \phi P_{0}\left(S_{0}\right)$ with: $|\phi\rangle=s \bar{s}$ and $\left|P_{0}\left(S_{0}\right)\right\rangle=X_{P_{0}, S_{0}}|u, d ; \mp\rangle+$ $Y_{P_{0}, S_{0}}|s, s ; \mp\rangle$. The two components $|u, d ; \mp\rangle$ and $|s, s ; \mp\rangle$ are separately shown.

$\overline{3}$ with $J^{P}=0^{+}$, totally antisymmetric in color, flavor and spin), with zero total spin and angular momentum. The quantum numbers of the $\phi S_{0}^{4}$ final state remain $I^{G}\left(J^{P C}\right)=$ $0^{-}\left(1^{--}\right)$, but, if we assume a structure like $[n s][\overline{n s}]$, where $n=u, d$, for the meson $S_{0}^{4}$, then both $\phi$ - and $\omega$-family contributions are now OZI-favored (see fig. 8).
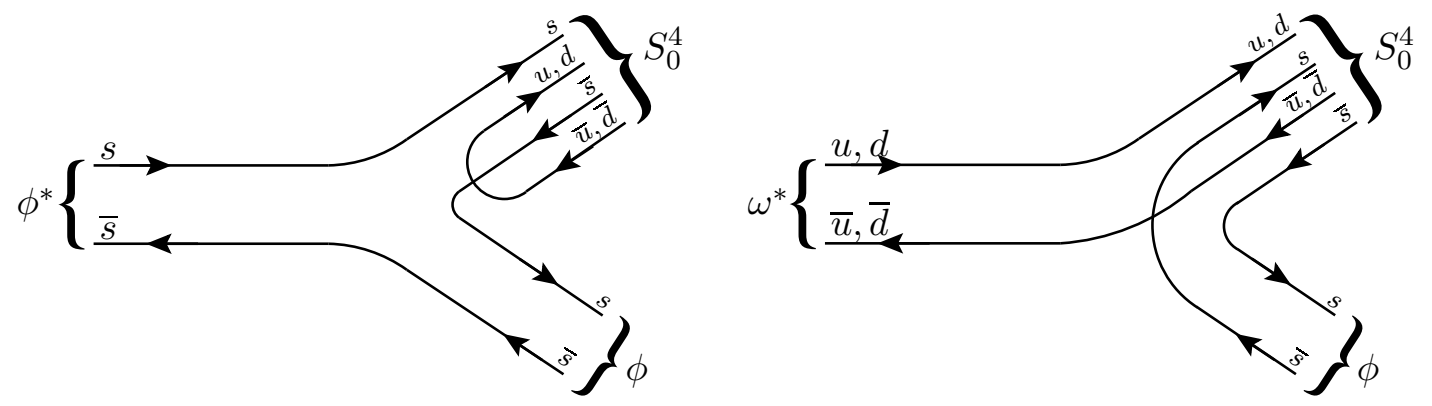

Figure 8: Feynman diagrams for the $\phi^{*}$ and $\omega^{*}$ contribution to the $\phi S_{0}^{4}$ final state.

To summarize, in the resonance region we consider three possibilities corresponding to different choices of the meson $M_{0}$ :

- $M_{0}=$ isospin zero, pseudoscalar meson $P_{0}$ :

$$
F_{\phi P_{0}}^{\mathrm{Res}}(q)=\sum_{V=\phi, \phi^{\prime}, \ldots} \frac{M_{V}^{2}}{e F_{V}} g_{\phi P_{0}}^{V} \frac{e^{i \Phi_{V P_{0}}}}{M_{V}^{2}-q^{2}-i \Gamma_{V} M_{V}} \quad s_{\mathrm{th}}^{0} \leq q^{2} \leq s_{\mathrm{asy}}^{P_{0}}
$$


- $M_{0}=$ isospin zero, scalar $q \bar{q}$ meson $S_{0}$ :

$$
F_{\phi S_{0}}^{\mathrm{Res}}(q)=\sum_{V=\phi, \phi^{\prime}, \ldots} \frac{M_{V}^{2}}{e F_{V}} g_{\phi S_{0}}^{V} \frac{e^{i \Phi_{V S_{0}}}}{M_{V}^{2}-q^{2}-i \Gamma_{V} M_{V}} \quad s_{\mathrm{th}}^{0} \leq q^{2} \leq s_{\mathrm{asy}}^{S_{0}}
$$

- $M_{0}=$ isospin zero, scalar tetraquark meson $S_{0}^{4}$ :

$$
F_{\phi S_{0}^{4}}^{\mathrm{Res}}(q)=\sum_{V=\phi, \phi^{\prime}, \ldots, \omega, \omega^{\prime}, \ldots} \frac{M_{V}^{2}}{e F_{V}} g_{\phi S_{0}^{4}}^{V} \frac{e^{i \Phi_{V S_{0}^{4}}}}{M_{V}^{2}-q^{2}-i \Gamma_{V} M_{V}} \quad s_{\mathrm{th}}^{0} \leq q^{2} \leq s_{\mathrm{asy}}^{S_{0}^{4}} .
$$

Relative phases $\Phi_{V M_{0}}$ have been included to account for possible re-scattering effects. In all these cases the isospin of the final state is zero, hence the theoretical threshold is $s_{\text {th }}^{0}=\left(3 M_{\pi}\right)^{2}$ [eq. (2.12)].

\subsubsection{Isovector $M_{I=1}$}

In case of isovector pseudoscalar $\left[I^{G}\left(J^{P C}\right)=1^{-}\left(0^{-+}\right)\right]$or scalar $\left[I^{G}\left(J^{P C}\right)=1^{-}\left(0^{++}\right)\right]$ meson $M_{1}$ the final state $\phi M_{1}$ (the subscript 1 is the isospin) has quantum numbers $I^{G}\left(J^{P C}\right)=1^{+}\left(1^{--}\right)$. It follows that only $\rho$-family contributions are expected. However, from the Feynman diagrams shown in fig. 9, constructed using for $\phi, P_{1}$, and $S_{1}$ the quark structures of eq. (2.13), we note that all these contributions are OZI-suppressed.

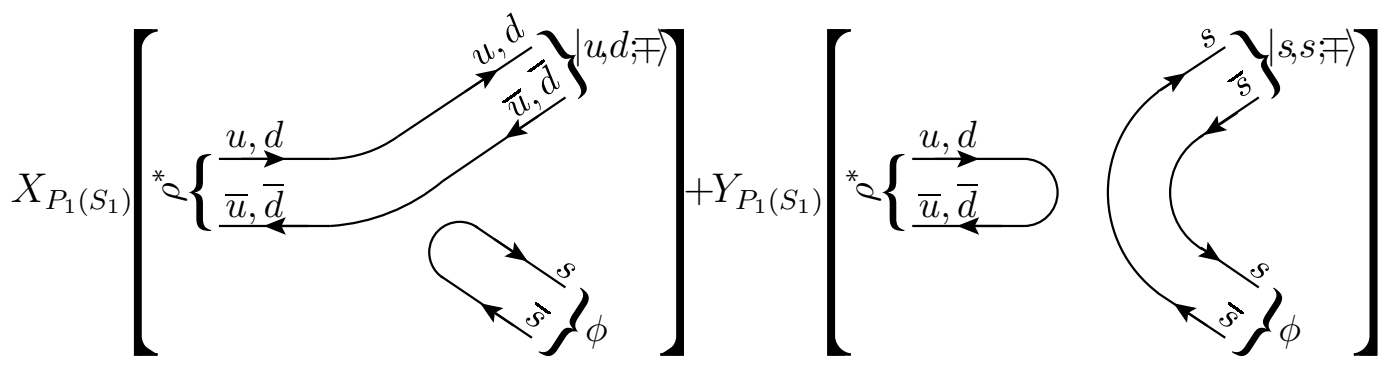

Figure 9: Feynman diagram for $\rho^{*} \rightarrow \phi P_{1}\left(S_{1}\right)$ with: $|\phi\rangle=s \bar{s}$ and $\left|P_{1}\left(S_{1}\right)\right\rangle=X_{P_{1}, S_{1}}|u, d ; \mp\rangle+$ $Y_{P_{1}, S_{1}}|s, s ; \mp\rangle$. The two components $|u, d ; \mp\rangle$ and $|s, s ; \mp\rangle$ are separately shown.

If we consider an isovector tetraquark scalar meson $S_{1}^{4}$, with structure $[n s][\overline{n s}](n=u, d$ as in case of isospin zero), then the additional hadronic field leads to OZI-favored coupling between intermediate $\rho$ recurrences and the final state $\phi S_{1}^{4}$ (see fig. 10).

To summarize, in the resonance region, two possible choices for $M_{1}$ are considered:

- $M_{1}=$ isospin one, pseudoscalar $P_{1}$ or scalar meson $S_{1}$ :

$$
F_{\phi M_{1}}^{\mathrm{Res}}(q)=\sum_{V=\rho, \rho^{\prime}, \ldots} \frac{M_{V}^{2}}{e F_{V}} g_{\phi M_{1}}^{V} \frac{e^{i \Phi_{V M_{1}}}}{M_{V}^{2}-q^{2}-i \Gamma_{V} M_{V}} \quad s_{\text {th }}^{1} \leq q^{2} \leq s_{\text {asy }}^{M_{1}}
$$

with $M_{1}=P_{1}, S_{1}$ and for all the couplings holds $g_{\phi M_{1}}^{V} \sim 0$, due to the OZI suppression (see fig. 9); 


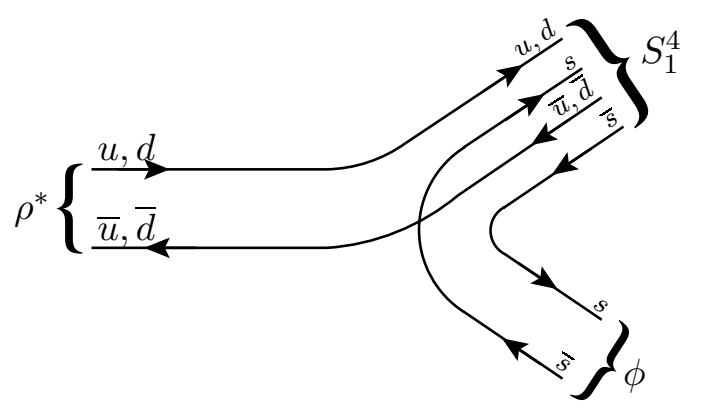

Figure 10: Feynman diagrams for the $\rho^{*}$ contribution to the $\phi S_{1}^{4}$ final state.

- $M_{1}=$ isospin one, scalar tetraquark meson $S_{1}^{4}$ :

$$
F_{\phi S_{1}^{4}}^{\mathrm{Res}}(q)=\sum_{V=\rho, \rho^{\prime}, \ldots} \frac{M_{V}^{2}}{e F_{V}} g_{\phi S_{1}^{4}}^{V} \frac{e^{i \Phi_{V S_{1}^{4}}}}{M_{V}^{2}-q^{2}-i \Gamma_{V} M_{V}} \quad s_{\mathrm{th}}^{1} \leq q^{2} \leq s_{\mathrm{asy}}^{S_{1}^{4}} .
$$

Again, the relative phases $\Phi_{V M_{1}}$ account for possible re-scattering effects and, having isospin one, the theoretical threshold is now $s_{\mathrm{th}}^{1}=\left(2 M_{\pi}\right)^{2}$.

\subsection{The asymptotic behavior}

A key point of this procedure to determine the parameters of the vector resonances is the knowledge of the tff time-like asymptotic behavior. For this purpose, we adopt the nominal power law predicted by QcR [4]. It follows that, as $q^{2}$ diverges, a hadronic ff must vanish like $\left(1 / q^{2}\right)$ to some power which linearly depends on the number of quark constituents and other quantum numbers of the hadrons under consideration. To describe the asymptotic behavior of the $\phi M_{I}$ tff's, we must also account for suppression factors related to the quark structure of the mesons, i.e.: the violation of the hadronic helicity conservation ${ }^{4}$ [7], which passes from zero, in the leptonic initial state, to one in the $\phi M_{I}$ final state $\left(\lambda_{M_{I}}=0\right.$, $\left|\lambda_{\phi}\right|=1$, where $\lambda_{\alpha}$ stands for the helicity of the particle $\alpha$ ), and the relative angular momentum between the quark and the antiquark in $M_{I}$. Since the asymptotic behavior does not depend on the isospin of the meson $M_{I}$, the following discussion holds for both $I=0$ and $I=1$.

${ }^{4}$ The virtual photon of the annihilation $e^{+} e^{-} \rightarrow \gamma^{*} \rightarrow \phi M_{I}$, at high energies, always has spin \pm 1 along the beam axis. Angular momentum conservation implies that the total angular momentum is 1 . In the center of mass frame: $\vec{p}_{\phi}=-\vec{p}_{M_{I}} \equiv \vec{p}$, it follows that

$$
\left|\frac{\vec{p}_{\phi} \cdot \vec{s}_{\phi}}{\left|\vec{p}_{\phi}\right|}-\frac{\vec{p}_{M_{I}} \cdot \vec{s}_{M_{I}}}{\left|\vec{p}_{M_{I}}\right|}\right|=\left|\lambda_{\phi}-\lambda_{M_{I}}\right|=\left|\frac{\vec{p} \cdot \vec{s}_{\text {tot }}}{|\vec{p}|}\right|=0,1
$$

where $\vec{s}_{\phi}$ and $\vec{s}_{M_{I}}$, and $\lambda_{\phi}$ and $\lambda_{M_{I}}$ are the spins and the helicities of the final mesons. But, since the hadronic helicity conservation requires: $\lambda_{\phi}+\lambda_{M_{I}}=0$, or $\lambda_{\phi}-\lambda_{M_{I}}=2 \lambda_{\phi}=-2 \lambda_{M_{I}}$, eq. (2.19) holds, in case of mesons, only if $\left|\lambda_{\phi}\right|=\left|\lambda_{M_{I}}\right|=0$. In the cases under consideration, the coupling $\phi \gamma^{*} M_{I}$ is described in terms of only one tff $F_{\phi M_{I}}\left(q^{2}\right)$ and this requires $\left|\lambda_{\phi}\right|=1$. Helicity zero would mean that the $\phi$ spin lies in the plane orthogonal to its 3-momentum, in this case we would need an additional degree of freedom (for instance: the azimuthal angle) and then an additional tff to describe this process. But since the tff is only one the $\phi$ spin must lie in 3-momentum direction, i.e. $\left|\lambda_{\phi}\right|=1$. 
As discussed in ref. [7], the cross section for the process $e^{+} e^{-} \rightarrow \phi M_{I}$ scaled by the $\mu^{+} \mu^{-}$ cross section goes asymptotically $\left(q^{2} \rightarrow \infty\right)$ as:

$$
\frac{\sigma_{\phi M_{I}}\left(q^{2}\right)}{\sigma_{\mu^{+} \mu^{-}}\left(q^{2}\right)} \propto\left(\frac{1}{q^{2}}\right)^{2\left(n_{H}-1\right)+n_{\lambda}+l_{q}} \quad \begin{aligned}
& n_{H}=\text { number of hadronic fields } \\
& n_{\lambda}=\left|\lambda_{M_{I}}+\lambda_{\phi}\right| \\
& \\
& l_{q}=q \bar{q} \text { relative angular momentum in } M_{I} .
\end{aligned}
$$

The asymptotic behavior of $\sigma_{\phi M_{I}}\left(q^{2}\right)$ [eq. 2.7)] depends on the tff, while the one of $\sigma_{\mu^{+} \mu^{-}}\left(q^{2}\right)$ is well-known:

$$
\sigma_{\phi M_{I}}\left(q^{2}\right) \underset{q^{2} \rightarrow \infty}{\propto}\left|F_{\phi M_{I}}\left(q^{2}\right)\right|^{2} \quad \sigma_{\mu^{+} \mu^{-}}\left(q^{2}\right) \underset{q^{2} \rightarrow \infty}{\propto} \frac{1}{q^{2}} .
$$

From previous equations we may extract the asymptotic behavior for the tff as:

$$
\left|F_{\phi M_{I}}\left(q^{2}\right)\right| \underset{q^{2} \rightarrow \infty}{\propto}\left(\frac{1}{q^{2}}\right)^{n_{H}+\frac{n_{\lambda}+l_{q}-1}{2}} .
$$

In the three cases investigated, we obtain the following asymptotic power laws:

- for $M_{I}=P_{I}:\left(n_{H}, n_{\lambda}, l_{q}\right)=(2,1,0)$, there are two hadronic fields in the final state (fig. 6), the hadronic helicity flips because $\lambda_{P_{I}}=0$ and $\lambda_{\phi}=1$ and, being the pseudoscalar $q \bar{q}$ state a $n^{2 S+1} L_{J}=1^{1} S_{0}$ element of the pseudoscalar flavor-SU(3) nonet, $l_{q}=0$ :

$$
\left|F_{\phi P_{I}}^{\text {asy }}\left(q^{2}\right)\right|=\left|F_{\phi P_{I}}^{\operatorname{Res}}\left(s_{\text {asy }}^{P_{I}}\right)\right|\left(\frac{s_{\text {asy }}^{P_{I}}}{q^{2}}\right)^{2} \quad q^{2}>s_{\text {asy }}^{P_{I}}
$$

- for $M_{I}=S_{I}:\left(n_{H}, n_{\lambda}, l_{q}\right)=(2,1,1)$, there are two hadronic fields in the final state (fig. 6) and the hadronic helicity flips $\left(\lambda_{S_{I}}=0\right)$, but the scalar $q \bar{q}$ state is an $n^{2 S+1} L_{J}=1^{3} P_{0}$ element of the scalar flavor-SU(3) nonet, hence $l_{q}=1$ :

$$
\left|F_{\phi S_{I}}^{\text {asy }}\left(q^{2}\right)\right|=\left|F_{\phi S_{I}}^{\mathrm{Res}}\left(s_{\mathrm{asy}}^{S_{I}}\right)\right|\left(\frac{s_{\mathrm{asy}}^{S_{I}}}{q^{2}}\right)^{\frac{5}{2}} \quad q^{2}>s_{\mathrm{asy}}^{S_{I}} ;
$$

- finally, for $M_{I}=S_{I}^{4}:\left(n_{H}, n_{\lambda}, l_{q}\right)=(3,1,1)$, due to the tetraquark structure of the scalar meson $S_{I}^{4}$, there are three hadronic fields in the final state (fig. \&), the hadronic helicity flips $\left(\lambda_{S_{I}^{4}}=0\right)$ and, having $S=L=0$, this is an $n^{2 S+1} L_{J}=1^{1} S_{0}$ element of the scalar flavor-SU(3) diquark nonet, hence $l_{q}=0$ (note that the quantum numbers do not refer to single quarks, but to the diquarks $[q q])$ :

$$
\left|F_{\phi S_{I}^{4}}^{\mathrm{asy}}\left(q^{2}\right)\right|=\left|F_{\phi S_{I}^{4}}^{\operatorname{Res}}\left(s_{\mathrm{asy}}^{S_{I}^{4}}\right)\right|\left(\frac{s_{\mathrm{asy}}^{S_{I}^{4}}}{q^{2}}\right)^{3} \quad q^{2}>s_{\mathrm{asy}}^{S_{I}^{4}} .
$$




\subsection{Analytic continuation in the $q^{2}$-complex plane}

As discussed in $\S 1.1$ a generic tff $F\left(q^{2}\right)$ is an analytic function in the complex $q^{2}$ plane with the cut $\left(s_{\text {th }}, \infty\right)$ (fig. 2). This property, together with the vanishing asymptotic behavior [eqs. (2.23 2.25)], allows one to use dispersion relations (DR) [8] to perform the analytic continuation of the tff's.

By applying the Cauchy theorem [8] to the integration path $\mathcal{C}$ of fig. 2, as the radius $\mathcal{R}$ diverges, we obtain the DR for the imaginary part:

$$
F\left(q^{2}\right)=\frac{1}{\pi} \int_{s_{\mathrm{th}}}^{\infty} \frac{\operatorname{Im}[F(s)]}{s-q^{2}} d s
$$

with $q^{2}<s_{\text {th }}$. Expression (2.26) states that real values of the tff $F\left(q^{2}\right)$, below the threshold $s_{\text {th }}$, may be computed by integrating its imaginary part over the cut.

To exploit directly the experimental data and the asymptotic behavior (2.23 2.25) for the modulus of the tff's in the time-like region, we use the DR for the logarithm [9]:

$$
\ln \left[F\left(q^{2}\right)\right]=\frac{\sqrt{s_{\mathrm{th}}-q^{2}}}{\pi} \int_{s_{\mathrm{th}}}^{\infty} \frac{\ln |F(s)|}{\left(s-q^{2}\right) \sqrt{s-s_{\mathrm{th}}}} d s \quad q^{2}<s_{\mathrm{th}},
$$

that connects the real values of the tff below the threshold $s_{\text {th }}$ to its modulus over the cut. The DR (2.27) is obtained by writing down the DR (2.26) for the function $\Psi(z)=\frac{\ln [F(z)]}{\sqrt{s_{\mathrm{th}}-z}}$ and it can be used in this form only if $F\left(q^{2}\right)$ has neither zeros nor poles on the physical sheet. The parameterizations (2.14 2.16) and (2.23 2.25) in the resonance and asymptotic regions guarantee the absence of singularities for the tff in physical sheet and allow one to use the DR for the logarithm (2.27).

Starting from the parameterizations for the modulus of the tff over the cut $\left(s_{\mathrm{th}}^{I}, \infty\right)$, by means of the DR (2.27), we obtained a parameterization also for the region below the threshold $s_{\text {th }}^{I}$ as:

$$
F_{\phi M_{I}}^{\mathrm{an}}\left(q^{2}\right)=\exp \left[\frac{\sqrt{s_{\mathrm{th}}^{I}-q^{2}}}{\pi}\left(\int_{s_{\mathrm{th}}^{I}}^{s_{\mathrm{asy}}^{M_{I}}} \frac{\ln \left|F_{\phi M_{I}}^{\mathrm{Res}}(s)\right| d s}{\left(s-q^{2}\right) \sqrt{s-s_{\mathrm{th}}^{I}}}+\int_{s_{\mathrm{asy}}^{M_{I}}}^{\infty} \frac{\ln \left|F_{\phi M_{I}}^{\mathrm{asy}}(s)\right| d s}{\left(s-q^{2}\right) \sqrt{s-s_{\mathrm{th}}^{I}}}\right)\right],
$$

with $M_{I}=P_{I}, S_{I}, S_{I}^{4}, I=0,1, q^{2}<s_{\text {th }}^{I}$ and where, in the first integral over the resonance region, we used expressions (2.14 2.18), while in the second, over the asymptotic region we used 2.232 .25$)$.

\subsection{The overall parameterization}

The overall parameterization for the tff's, which covers the whole real $q^{2}$ axis (in principle the whole complex $q^{2}$ plane), has the threefold expression (see fig. 11):

$$
F_{\phi M_{I}}\left(q^{2}\right)=\left\{\begin{array}{lll}
F_{\phi M_{I}}^{\mathrm{an}}\left(q^{2}\right) & q^{2} \leq s_{\mathrm{th}}^{I} & {[\text { eq. (2.28)] }} \\
F_{\phi M_{I}}^{\mathrm{Res}}\left(q^{2}\right) & s_{\mathrm{th}}^{I}<q^{2} \leq s_{\mathrm{asy}}^{M_{I}} & {[\text { eqs. (2.14)-(2.18)] }} \\
F_{\phi M_{I}}^{\mathrm{asy}}\left(q^{2}\right) & q^{2}>s_{\mathrm{asy}}^{M_{I}} & {[\text { eq. (2.23)-(2.25)] }}
\end{array}\right.
$$




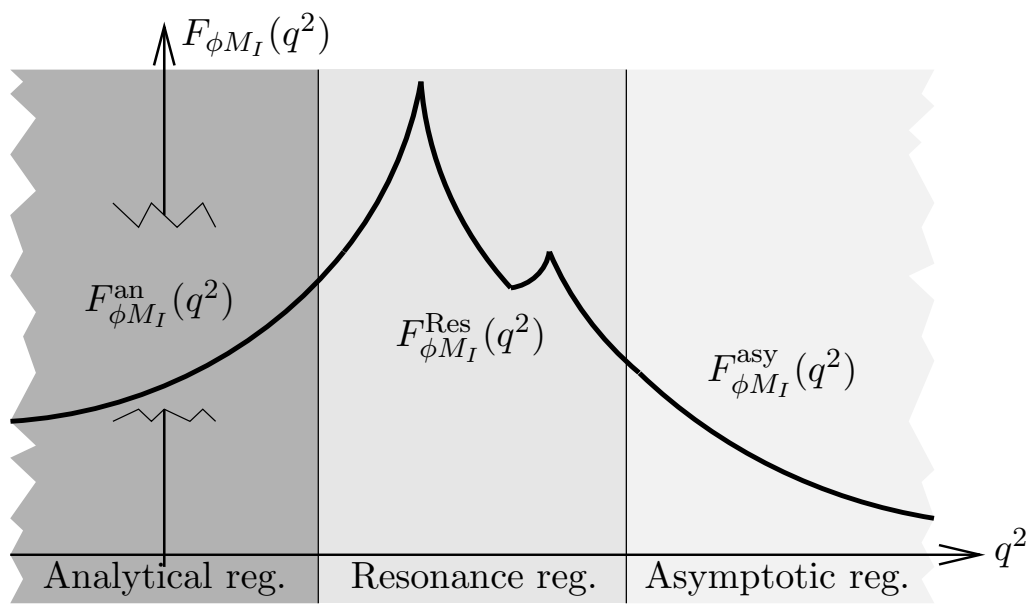

Figure 11: Schematic representation of the three energy regions used to parameterize the tff's.

with $M_{I}=P_{I}, S_{I}, S_{I}^{4}$ and $I=0,1$.

Figure 11 shows the regions in which the real $q^{2}$ axis has been divided to parametrize the tff's. Starting from the right there are:

- the asymptotic region, in light-gray, where the QcR power law has been used;

- the resonance region, in gray, where the tff's are parametrized as a sum of resonant contributions;

- the analytic region, in dark-gray, where the tff's are obtained through the DR's.

\section{3. $\chi^{2}$ definition}

\subsection{Free parameters and fixed values}

The free parameters of the expression (2.29) are all located in the resonant part of the definition $F_{\phi M_{I}}^{\text {Res }}\left(q^{2}\right)$. Different choices may be done about masses and widths of the contributing vector resonances. For the leading contributions, i.e.: the $\phi(1020)$ in case of $M_{0}=P_{0}, S_{0}$, the $\omega(782)$ in case of $M_{0}=S_{0}^{4}$ and the $\rho^{0}(770)$ in case of $M_{1}=P_{1}, S_{1}, S_{1}^{4}$, fixed values of masses and widths may be used, because many of these quantities are known with a high degree of accuracy. Other masses and widths, namely those of $\phi-, \omega$ - and $\rho$ recurrences, being poorly known, are left free, indeed one of the aims of the analysis is a better determination of their values.

Concerning the couplings, while some values of $F_{V}$ may be extracted from data on decay rates $\Gamma\left(V \rightarrow e^{+} e^{-}\right)$and then they are assumed as fixed, all the other couplings $F_{V}$ and $g_{\phi M_{I}}^{V}$, and the relative phases $\Phi_{V M_{I}}$ are considered as free parameters.

Finally, also the threshold energies $s_{\text {asy }}^{M_{I}}$, from which the asymptotic behavior is assumed, are left free. 


\subsection{Constraints}

Two kinds of conditions may be used to fix the free parameters of the procedure: the experimental and theoretical constraints.

In general, the experimental constraints come from data for three different processes:

- the radiative decay $\phi \rightarrow M_{I} \gamma$ gives the real value of the tff at $q^{2}=0$;

- the conversion decay $\phi \rightarrow M_{I} e^{+} e^{-}$covers the interval $\left(2 m_{e}\right)^{2} \leq q^{2} \leq\left(M_{\phi}-M_{M_{I}}\right)^{2}$, it gives the real value of the tff below $s_{\text {th }}^{I}$ and its modulus above this threshold;

- the annihilation $e^{+} e^{-} \rightarrow \phi M_{I}$ provides a measurement of the modulus of the tff above the physical threshold, i.e. for $q^{2} \geq\left(M_{\phi}+M_{M_{I}}\right)^{2}$.

The theoretical condition requires analyticity for the tff, imposing the continuity of its first derivative across the threshold $s_{\text {th }}^{I}$, by means of the DR (2.27), i.e., it forces the identity:

$$
\left.\frac{1}{F_{\phi M_{I}}^{\mathrm{Res}}} \frac{d F_{\phi M_{I}}^{\mathrm{Res}}}{d s}\right|_{s_{\mathrm{th}}^{I}}=\lim _{\epsilon \rightarrow 0^{+}} \frac{1}{2 \sqrt{\epsilon}} \int_{s_{\mathrm{th}}^{I}}^{\infty} \frac{\left(s_{\mathrm{th}}^{I}-s+\epsilon\right) \ln \left|F_{\phi M_{I}}(s)\right|}{\sqrt{s-s_{\mathrm{th}}^{I}}\left(s-s_{\mathrm{th}}^{I}+\epsilon\right)^{2}} d s .
$$

This condition represents a further constraint which concerns both the resonance and the asymptotic part of the parameterization (2.29). Note that the DR for the logarithm (2.27) automatically ensures continuity for the tff (zero derivative) across $s_{\mathrm{th}}^{I}$.

\section{$3.3 \chi^{2}$ expression}

Following the previous discussion we define a $\chi^{2}$ with two contributions:

$$
\chi_{M_{I}}^{2}=\chi_{M_{I}, \exp }^{2}+\chi_{M_{I}, \text { theory }}^{2} .
$$

The experimental contribution is defined as:

$$
\chi_{M_{I}, \exp }^{2}=\sum_{j}\left[\frac{\left|F_{\phi M_{I}}\left(s_{j}^{M_{I}}\right)\right|-F_{j}^{M_{I}}}{\delta F_{j}^{M_{I}}}\right]^{2},
$$

where $\left\{s_{j}^{M_{I}}, F_{j}^{M_{I}} \pm \delta F_{j}^{M_{I}}\right\}$ is the total set of data on the $\phi M_{I}$ tff, as we have discussed in the previous sections, these data may be extracted from decay rates and cross sections of different processes which involve the mesons $\phi$ and $M_{I}$.

The theoretical contribution, which forces the continuity of the first derivative of the tff across the threshold $s_{\mathrm{th}}^{I}$, has the form [eq. (3.1)]:

$$
\chi_{M_{I}, \text { theory }}^{2}=\tau \cdot\left[\left.\frac{1}{F_{\phi M_{I}}^{\mathrm{Res}}} \frac{d F_{\phi M_{I}}^{\mathrm{Res}}}{d s}\right|_{s_{\mathrm{th}}^{I}}-\lim _{\epsilon \rightarrow 0^{+}} \frac{1}{2 \sqrt{\epsilon}} \int_{s_{\mathrm{th}}^{I}}^{\infty} \frac{\left(s_{\mathrm{th}}^{I}-s+\epsilon\right) \ln \left|F_{\phi M_{I}}(s)\right|}{\sqrt{s-s_{\mathrm{th}}^{I}}\left(s-s_{\mathrm{th}}^{I}+\epsilon\right)^{2}} d s\right]^{2},
$$

where $\tau$ is a free parameter. Since the theoretical condition has to be exactly verified in order to satisfy the analyticity requirement, the values of $\tau$ can be chosen large enough

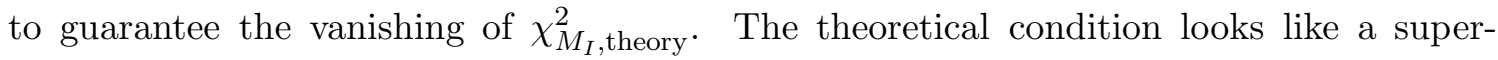
convergence relation, indeed it constrains the value of an integral of the tff over the cut and it represents a crucial constraint in the resonance region, below the physical threshold, where there are no data. 


\section{Possible aims}

\subsection{Looking for masses, widths and coupling constants}

The main aim of this analysis procedure is to provide a practical tool, based on theory and data, to find masses, widths and coupling constants of vector mesons, like $\phi-, \omega$ - and $\rho$-recurrences, that are still poorly known. The key points of this method, which make it a step forward with respect to the usual fit procedures, are:

- the analysis is performed in terms of tff's instead of single observables like: decay rates and cross sections, this allows to use at the same time, as measurements of the same quantity in different energy regions, data coming from different processes;

- by using an analytic continuation procedure based on DR's, additional information about the quark structure of the mesons, coming from the QcR power law used for the asymptotic behavior, may be included in the computation;

- thanks to overall validity of the parameterization, we may analyze resonances lying in energy regions not experimentally accessible, like the unphysical region $\left[\left(M_{\phi}-\right.\right.$ $\left.\left.M_{M_{I}}\right)^{2},\left(M_{\phi}+M_{M_{I}}\right)^{2}\right]$, or at energies hardly reachable, e.g. near thresholds.

In other words, the constraints from data and theory shape, through the analyticity requirement, the tff's at every value of $q^{2}$.

\subsection{Investigating quark structures}

Since all the three steps of this procedure: parameterization in the resonance region $(\$ 2.3)$, definition of the asymptotic behavior ( $\$ 2.4$ ) and extension below the threshold $s_{\text {th }}^{I}(\$ 2.5)$, depend on the assumed quark structure for the meson $M_{I}$, we may exploit this method to test the agreement with data of different possible structures and then to gain information on the nature of the meson $M_{I}$.

The most interesting cases are those of scalar mesons $S_{I}$ and $S_{I}^{4}$. In fact we noted that by passing from the $q \bar{q}$ to the $[q q][\overline{q q}]$ structure, the tff parameterization changes drastically in all the three regions.

In particular for $I=0$, the tetraquark hypothesis for the scalar meson implies additional $\omega$-family contributions in the resonance region and a slightly faster vanishing asymptotic behavior, the power goes from $5 / 2$ to 3 .

In case of $I=1$ the situation appears more drastic, in fact while the conversion $\phi \gamma S_{1}$, with a $q \bar{q}$-scalar meson is OZI-suppressed (fig. 9), the same conversion $\phi \gamma S_{1}^{4}$, but with a tetraquark $S_{1}^{4}$, should be, instead, OZI-allowed (fig. 10).

\section{Conclusion}

We have defined a general procedure which, combining: the description of the tff's in terms of vector meson propagators, the QcR asymptotic power law, and the analyticity requirement by means of DR's, provides a parameterization for the tff's valid in the whole 
$q^{2}$-real axis. The experimental and theoretical constraints, that individually concern only certain energy regions, have been "propagated" to all values of $q^{2}$.

The application of this procedure may be twofold.

By assuming as known the quark structure of the mesons under consideration, we can look for vector resonances, i.e. excited states of the $\phi(1020), \omega(782)$ and $\rho^{0}(770)$, which couple with the studied final state.

By exploiting the fact that this procedure is strongly dependent on the quark structure of the final mesons, we may test how well different hypotheses about the this structure agree with data.

\section{Acknowledgments}

I warmly thank Rinaldo Baldini, Gino Isidori, Lia Pancheri, Mike Sokoloff, and Adriano Zallo for precious and instructive discussions on the subject of this work.

\section{References}

[1] B. Di Micco [KLOE Collaboration], arXiv:hep-ex/0410072;

V. Druzhinin [BABAR Collaboration], arXiv:hep-ex/0601020.

[2] O. Gayou et al. [Jefferson Lab Hall A Collaboration], Phys. Rev. Lett. 88 (2002) 092301 [arXiv:nucl-ex/0111010].

[3] L. G. Landsberg, Phys. Rept. 128 (1985) 301.

[4] V. A. Matveev, R. M. Muradian and A. N. Tavkhelidze, Lett. Nuovo Cim. 7, 719 (1973);

S. J. Brodsky, G. R. Farrar, Phys. Rev. D 11 (1975) 1309;

S. J. Brodsky and B. T. Chertok, Phys. Rev. D 14 (1976) 3003.

[5] G. Zweig, CERN report S419/TH412 (1964), unpublished;

S. Okubo, Phys. Lett, 5 (1963) 165;

I. Iizuka, K. Okuda, O. Shito, Prog. Theor. Phys. 35 (1966) 1061.

[6] R. L. Jaffe, Phys. Rept. 409 (2005) 1 [Nucl. Phys. Proc. Suppl. 142 (2005) 343] [arXiv:hep-ph/0409065] and rferrences therein.

[7] S. J. Brodsky, G. P. Lepage, Phys. Rev. D 24 (1981) 2848. S. J. Brodsky and G. F. de Teramond, Phys. Lett. B 582 (2004) 211 [arXiv:hep-th/0310227].

[8] See for instance: E.C. Tichmarsch, The theory of functions, London, Oxford University Press, 1939.

[9] B. V. Geshkenbein, Yad. Fiz. 9 (1969) 1232. 